retrospective, have not defined IBS-D according to accepted diagnostic criteria, or have included patients with chronic diarrhoea in the analysis. We have examined this issue in a well-characterised cohort of patients with rigorously defined IBS-D.

Methods This was a prospective cross-sectional survey conducted among consecutive patients with IBS-D attending Gastroenterology clinics in two hospitals in Sheffield and Leeds, UK. All patients underwent 23-seleno-25-homo-tauro-cholic acid (SeHCAT) scanning according to local protocol, with a retention of $<15 \%$ at day 7 used to confirm BAD. The degree of BAD was classed as severe if retention $<5 \%$, moderate if $5.0>9.9 \%$, and mild if $10.0>14.9 \%$. Presence of IBS-D was defined according to the Rome III criteria. Patients with other known risk factors for $\mathrm{BAD}$, including previous cholecystectomy, terminal ileal Crohn's disease, terminal ileal resection, pelvic or abdominal radiotherapy, coeliac disease, or microscopic colitis, were excluded. Participants completed the patient health questionnaire-15, a validated somatisation score, and the hospital anxiety and depression score. Demographic data, including age, gender, lifestyle, and body mass index (BMI) were collected. The effect of all these factors on presence or absence of BAD was examined by multivariate logistic regression analysis, with results expressed as odds ratios (ORs) with 99\% confidence intervals.

Results This is an interim analysis of an ongoing study. In total, 51 patients with IBS-D according to the Rome III criteria have been recruited to date (37 (72.5\%) female, mean age 47.0 years). In total, $14(27.5 \%)$ were found to have BAD following $\mathrm{SeH}$ CAT scanning. Of these, nine (17.6\%) had severe BAD, four moderate, and one mild. Mean age, BMI, anxiety, depression, and somatisation scores were not significantly different among those with, compared with those without, BAD. No predictors of presence of $\mathrm{BAD}$ were identified following multivariate logistic regression.

Conclusion Our data suggest that more than one-in-four IBS-D patients, if investigated, have definite evidence of BAD. In the majority, this is severe. Failure to investigate patients to exclude $\mathrm{BAD}$ as an underlying cause of symptoms compatible with IBS-D results in misdiagnosis and a failure to institute effective therapy, in the form of bile acid sequestrants. This suggests that future IBS management guidelines should advocate diagnostic testing to exclude BAD before a diagnosis of IBS-D is made.

Disclosure of Interest None Declared.

\section{PTH-107 A PRELIMINARY STUDY OF THE EFFECTS OF OBETICHOLIC ACID, A FARNESOID X RECEPTOR AGONIST, IN PATIENTS WITH CHRONIC DIARRHOEA SECONDARY TO CROHN'S ILEAL DISEASE}

${ }^{1} \mathrm{JD}$ Nolan*, ${ }^{1} \mathrm{C}$ Vassie, ${ }^{1}$ IM Johnston, ${ }^{2} \mathrm{D}$ Shapiro, ${ }^{1} \mathrm{JR}$ Walters. ${ }^{1}$ Gastroenterology, Imperial College London, London, UK; ${ }^{2}$ Pharmaceuticals, Intercept Pharmaceuticals, San Diego, USA

\subsection{6/gutjnl-2014-307263.553}

Introduction Chronic diarrhoea occurs frequently as a result of excess faecal bile acid (BA) loss. Secondary bile acid diarrhoea (SBAD) is common in Crohn's disease with ileal inflammation and/or resection. The normal ileum produces Fibroblast Growth Factor 19 (FGF19) in response to BA absorption and farnesoid $\mathrm{X}$ receptor (FXR) activation. FGF19 acts as a hormonal regulator of hepatic BA synthesis. We showed previously in 10 patients with primary bile acid diarrhoea, diagnosed by $7 \mathrm{~d}$ SeHCAT retention $<10 \%$, that the semi-synthetic BA and potent FXR agonist obeticholic acid (OCA) significantly increased low
FGF19 levels producing significant clinical improvement. We aimed to see if these findings could be extended to patients with SBAD due to Crohn's and in idiopathic diarrhoea controls.

Methods Out of 32 patients recruited to this pilot trial, 8 SBAD patients $(6 \mathrm{~F}: 2 \mathrm{M}$, median age 45 , ileal resection $0-48 \mathrm{~cm}$, median $22.5 \mathrm{~cm}$, and/or SeHCAT <11\%), and 7 controls (2F:5M, SeHCAT $16-35 \%$, median $25 \%$ ) received OCA $25 \mathrm{mg}$ daily for $2 \mathrm{w}$ after a $2 \mathrm{w}$ run-in period. BA sequestrants were discontinued. Symptoms were recorded and a stool index calculated from frequency, stool form and loperamide use. On the first and last days of OCA therapy, blood samples were assayed for FGF19, total BA levels and the BA precursor, $7 \alpha \mathrm{OH}-4$-cholesten-3-one (C4) in fasting and for 6h after OCA and meals.

Results In the SBAD group, 7 out of 8 patients showed positive but variable changes in stool form and stool index (both $\mathrm{p}=$ 0.07 , Wilcoxon). Pain frequency $(\mathrm{p}=0.05)$ and severity $(\mathrm{p}=$ $0.07)$ improved. Ileal resection length was related to the change in stool number $(r=0.78, p=0.01$, Spearman), index $(r=$ $0.63, p=0.05)$ and urgency $(r=0.68, p=0.03)$ so that those with the smallest resections had the greatest improvements. Increases in FGF19 fasting and post-prandial levels were relatively small except in 2 patients, but were associated with improvements in urgency $(\mathrm{r}=0.93, \mathrm{p}<0.01)$. The reductions in post-prandial BA response $(\mathrm{p}=0.01)$, fasting and peak BA values were significantly greater in those with shorter resections. C4 was related inversely to FGF19 and positively to the resection length. By contrast in the diarrhoea controls, there were no significant changes in clinical symptoms or FGF19. However BA responses were lower $(p=0.03)$ and significant relationships between FGF19 and BA responses were found.

Conclusion This pilot study has shown that OCA produces clinical benefit in many patients with chronic diarrhoea including those with SBAD, particularly with short resections, but not in idiopathic controls. Further trials are warranted.

Disclosure of Interest None Declared.

\section{PTH-108 SEHCAT: NICE OR NOT NICE?}

KL Woolson*, H Sherfi, T Sulkin, J Palmer, IA Murray. Gastroenterology, Royal Cornwall Hospital, Truro, UK

\subsection{6/gutjnl-2014-307263.554}

Introduction Bile acid malabsorption (BAM) is increasingly recognised as the underlying diagnosis in many patients with D-IBS and Crohn's disease, and SeHCAT testing has greatly increased. The 2012 NICE consultation document ${ }^{1}$ acknowledges lack of evidence of cost effectiveness and advocates trial of treatment with bile acid sequestrants (BAS) rather than SeHCAT for Crohn's patients, but often these are poorly tolerated and the response equivocal. We review our experience of SeHCAT testing and review it with respect to NICE.

Methods Retrospective review of 121 consecutive patients who had SeHCAT performed between April 2009 and December 2012. Patient demographics, associated diseases (Crohn's disease, right hemicolectomy, radiotherapy, HIV, microscopic colitis, coeliac disease, vagotomy and pyloroplasty, Graves disease, intestinal

\begin{tabular}{lllll}
\multicolumn{1}{l}{ Abstract PTH-108 Table 1} \\
\hline & Sensitivity & Specificity & PPV & NPV \\
\hline Crohn's & 0.75 & 0.60 & 0.90 & 0.32 \\
Right hemicolectomy & 0.88 & 0.59 & 0.97 & 0.26 \\
\hline
\end{tabular}


bypass, cholecystectomy), symptoms, previous tests and outcomes of BAS were reviewed. Logistic regression was performed to determine predictors of BAM.

Results Patient age range was $18-85$ years, median 50 years with the majority female $(76 ; 63 \%)$. Of the patients investigated with SeHCAT scan, $78 \%$ had had a colonoscopy, 33\% an OGD and $21 \%$ a CT scan.

Only Crohn's disease and right hemicolectomy were significantly associated with BAM. The frequency and nature (steatorrhoea or watery diarrhoea) of the stool was not significantly correlated with BAM.

The number of SeHCAT tests increased from 2 in 2009 to 62 in 2012. $57(47 \%)$ had a positive scan of who $83 \%$ were given BAS post-test and of these $52 \%$ had a good response to therapy, $23 \%$ didn't respond and 10\% couldn't tolerate the BAS. $14 \%$ of patients had a trial of therapy pre-test;38\% of these responded to therapy. $29 \%$ of patients given BAS weren't seen after treatment so response is unknown. Unsurprisingly of those with a negative SeHCAT scan $(n=63)$ only one patient had a partial response to treatment. Of these 6 were given treatment prior to their test. Only one had Crohn's disease and none had a right hemicolectomy so the trial of treatment in most was unnecessary.

Conclusion SeHCAT was often performed after many other investigations for diarrhoea. NICE guidelines suggest SeHCAT scan should be considered early in the investigation of chronic diarrhoea.

Of the SeHCAT scans performed, 57\% were positive and could have prevented invasive tests if performed earlier. For patients with Crohn's disease or right hemicolectomy sensitivity and PPV was sufficiently high to warrant treatment without testing as per NICE.

\section{REFERENCE}

1 SeHCAT (Tauroselcholic [75Selenium] acid) for the investigation of bile acid malabsorption (BAM) and measurement of bile acid pool loss. http://guidance.nice. org.uk/DT/8

Disclosure of Interest None Declared.

\section{PTH-109 MANAGEMENT OF BILE ACID MALABSORPTION (BAM) WITH LOW FAT DIETARY INTERVENTIONS}

'L Watson*, ${ }^{2} \mathrm{~A}$ Lalji, ${ }^{3} \mathrm{~S}$ Bodla, ${ }^{2} \mathrm{~J}$ Andreyev, ${ }^{1} \mathrm{C}$ Shaw. ${ }^{1}$ Department of Nutrition and Dietetics, The Royal Marsden NHS Foundation Trust, London, UK; ${ }^{2}$ GI Unit, The Royal Marsden NHS Foundation Trust, London, UK; ${ }^{3}$ Statistics, The Royal Marsden NHS Foundation Trust, London, UK

\subsection{6/gutjnl-2014-307263.555}

Introduction BAM is the unrecognised cause for loose stool for 500,000 people in the UK. It is increasingly recognised as a potential cause of distressing gastrointestinal (GI) symptoms after cancer treatment. This study aims to evaluate the efficacy of low fat dietary interventions in the management of BAM.

Methods Patients with new onset GI symptoms after cancer treatment and a 7 day 23-selena-25-homochololytaurine (SeHCAT) scan $<20 \%$, were included in a prospective service evaluation. Patients were advised on a low fat dietary intervention by a Registered Dietitian, which aimed to provide $20 \%$ of total energy from fat. Patients rated their GI symptoms using a 10 point numerical rating scale, and completed 7 day dietary diaries, before and after dietary intervention. The dietary diaries were analysed using the dietplan6 dietary analysis programme. Significance of changes in symptom scores were analysed using
Wilcoxon signed-ranks test, change in dietary fat intake using a paired t-test.

Results 40 patients (20 male, 20 female) with a median age 61 (range 22-90) years were recruited. The cancer diagnoses were GI (28\%), gynaecological (30\%), urological (30\%) and other (12\%). $7.5 \%$ had borderline BAM (15-20\% 7 day retention), $25 \%$ mild BAM (10-15\% retention), $17.5 \%$ moderate $(5-10 \%$ retention) and $50 \%$ severe ( $<5 \%$ retention). $62.5 \%$ of patients were taking a bile acid sequestrant. Symptoms reported were urgency $(83 \%)$, bloating (43\%), increased frequency (43\%), lack of control (40\%), abdominal pain (38\%), nocturnal defaecation (28\%), incomplete evacuation (25\%) and greasy/pale stools (23\%). After dietary intervention, the mean scores for all symptoms decreased. There was a significant reduction in mean ratings for urgency, bloating, lack of control, bowel frequency ( $\mathrm{p}=$ $<0.01$ ), flatulence, abdominal pain, greasy/pale stool and abdominal gurgling $(\mathrm{p}=<0.05)$. Mean dietary fat intake reduced from $62.3 \mathrm{~g}$ of fat before dietary intervention to $42.2 \mathrm{~g}$ of fat after intervention $(\mathrm{p}=<0.01)$. There was no statistically significant change in dietary fibre intake.

Conclusion The use of low fat dietary interventions in patients with a SeHCAT scan $<20 \%$ leads to clinically important improvement in GI symptoms and should be widely used.

Disclosure of Interest None Declared.

\section{PTH-110 FACTORS PREDICTIVE OF BILE ACID DIARRHOEA AND LONG TERM TREATMENT OUTCOMES}

M Kurien*, JT Gleeson, C Osborne, L Messham, DS Sanders. Department of Gastroenterology, Royal Hallamshire Hospital, Sheffield, UK

\subsection{6/gutjnl-2014-307263.556}

Introduction Bile acid diarrhoea (BAD) is a recognised cause of chronic diarrhoea, however detection remains sub-optimal. Knowledge of factors predictive of BAD could help improve detection. This study evaluates factors predictive of BAD (7 day SeHCAT retention $<10 \%$ ) and determines long treatment outcomes in those instigated on medical therapy.

Methods 515 patients underwent a SeHCAT test in a University hospital (2001-2012). Of these 41\% (210/515) had evidence of BAD. Demographic data, clinical and biochemical indices were collected in all patients tested. Binary logistic regression was then used to determine factors predictive of BAD. 107 of the 210 (51\%) patients with BAD were diagnosed between 20012009 and commenced on bile acid sequestrants. ${ }^{1}$ In March 2013, these patients $(n=107)$ were re-contacted and reassessed either in a gastroenterology clinic or via telephone consultation, determining their bowel frequency, current symptoms, response to bile acid sequestrants and whether on continuing treatment. Comparisons were made in pre and post treatment variables using a paired sample t-test.

Results Binary Logistic regression identified only terminal ileal Crohn's, terminal ileal resection, previous small bowel surgery and cholecystectomy as predictors of BAD ( $p<0.0001)$. With regards to follow up, 54\% (58/107) of patients (median age 57 years, range 29-74 years, 42 females) were contactable and agreeable to follow up assessment. The mean time since diagnosis of BAM was 7.1 years, with a mean result of $3.6 \%$ at diagnosis. $38 \%(22 / 58)$ of patients were still on bile acid sequestrants at follow up, with $28 \%$ using alternative anti-diarrhoeal agents. In those who were on bile acid sequestrant therapy, mean stool frequency decreased from 7.3 stools per day to 3.9 ( $\mathrm{p}<$ $0.0001)$. The $34 \%(20 / 58)$ of patients not receiving medical 